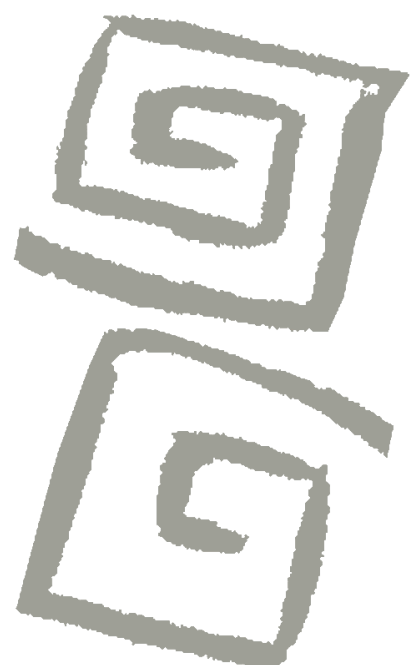

\title{
Mortalidad por mesotelioma en Argentina, 1980-2013
}

\author{
Mesothelioma mortality in Argentina, \\ 1980-2013
}

Andrés Trotta ${ }^{1}$, Vilma Sousa Santana², Marcio Alazraqui ${ }^{3}$

${ }^{1}$ Doctor en Salud Colectiva. Director, Maestría en Epidemiología, Gestión y Políticas de Salud, Instituto de Salud Colectiva, Universidad Nacional de Lanús, Argentina. $\bowtie$ (iD)

${ }^{2}$ Doctora en Epidemiología. Profesora Asociada III, Instituto de Saúde Coletiva, Universidade Federal da Bahia, Brasil. $\triangle$ (iD)

${ }^{3}$ Doctor en Salud Colectiva. Director, Doctorado en Salud Colectiva, Instituto de Salud Colectiva, Universidad Nacional de Lanús, Argentina. $\bowtie$ iD
RESUMEN Se estimó la tasa de mortalidad por mesotelioma y su distribución sociodemográfica y temporal en Argentina para el período 1980-2013 con datos del Sistema de Estadísticas Vitales del Ministerio de Salud de la Nación. Se encontraron 3.259 defunciones por mesotelioma, lo que resulta en una tasa de mortalidad estandarizada por edad de 3,1/1.000.000 en 1980 y de 5,7/1.000.000 en 2013, con un aumento promedio del $84,1 \%$ en 34 años. El incremento de la tendencia fue más claro a partir de 1997. En todos los años, la tasa de mortalidad fue mayor en hombres que en mujeres. Los resultados sugieren antecedentes de exposición al asbesto en el pasado. Aparentemente, la exposición predominante fue la ocupacional, más común entre los hombres que concentraron los casos. Se recomiendan acciones dirigidas a hacer más efectiva la prohibición ya en vigor y la vigilancia en salud orientada a los ambientes de trabajo, trabajadores previamente expuestos y la población en general.

PALABRAS CLAVES Mesotelioma; Mortalidad; Estudios de Series Temporales; Argentina.

ABSTRACT Mesothelioma mortality and its socio-demographic and temporal patterns in Argentina from 1980 to 2013 were estimated using data from death certificates obtained from the Vital Statistics System of Argentina's National Ministry of Health. There were 3,259 mesothelioma deaths corresponding to an age-adjusted mortality of $3.1 / 1,000,000$ in 1980 and 5.7/1,000,000 in 2013, an average increase of $84.1 \%$ in 34 years. This raising trend became clearer after 1997. Males had higher mortality estimates compared with women in every year of the series; these findings suggest past exposure to asbestos. It is plausible that the asbestos exposure was mostly occupational, which is more common among men. Actions related to reinforcing the asbestos ban already in place and strengthening health surveillance directed at workplaces, previously exposed workers, and the population in general are recommended.

KEY WORDS Neoplasms; Mesothelioma; Mortality; Time Series Studies; Argentina. 


\section{INTRODUCCIÓN}

El mesotelioma es un tumor maligno asociado a la exposición al asbesto, mineral fibroso ampliamente utilizado con fines $\mathrm{CO}^{-}$ merciales en todo el mundo y clasificado como cancerígeno tipo I por la International Agency for Research on Cancer (IARC) ${ }^{(1)}$. Este cáncer, de origen ocupacional y/o ambiental, es evitable, por lo que la Organización Mundial de la Salud (OMS) ha recomendado que los gobiernos promuevan la sanción de legislación para la prohibición total o parcial de la extracción, producción y uso de todas las formas de asbesto, las que ya fueron implementadas en 54 países ${ }^{(2)}$, incluso en Argentina, donde además de esta medida, las acciones de vigilancia de cancerígenos propuestas por el Ministerio de la Salud incluyen el monitoreo epidemiológico por medio de indicadores como la mortalidad por enfermedades relacionadas a estos factores de riesgo $^{(3)}$. Específicamente para el asbesto, la mortalidad por mesotelioma es uno de los indicadores más utilizados y relevantes para el monitoreo de los efectos del asbesto sobre la salud y también del impacto de las acciones de prevención ${ }^{(4)}$, aunque este tumor se asocie a un largo período de latencia.

Las estimaciones de mortalidad por mesotelioma varían ampliamente entre países y regiones del mundo. En un estudio con datos de 83 países, para el período de 1994 a 2008, se identificaron 92.253 defunciones, lo que implica una tasa de mortalidad estandarizada por edad de 4,2/1.000.000 para el total de la población de esos países. Las tasas más elevadas fueron las del Reino Unido $(17,8 / 1.000 .000)$, Australia (16,5/1.000.000) e Italia $(10,3 / 1.000 .000)^{(5)}$. En Europa, entre 36 países con registros de defunciones por mesotelioma, se identificaron 71.686 casos entre 1994-2010, y se estimó un riesgo medio de muerte de 7,7/1.000.000 para la población general en el período referido ${ }^{(6)}$. Solo en Gran Bretaña e Irlanda del Norte se registraron 13.517 defunciones entre 1994 y 2008 , lo que corresponde a una tasa de mortalidad bruta de 17,8/1.000.000 ${ }^{(7)}$. En Bélgica, en el mismo período, se registraron 1.467 defunciones por mesotelioma y se estimó una mortalidad estandarizada por edad de 9,6/1.000.000(8). En regiones con menor desarrollo económico como en América del Sur, los estudios epidemiológicos sobre el mesotelioma son escasos y las estimaciones sugieren que existe un subregistro de $\operatorname{casos}^{(9)}$. Brasil es el tercer productor mundial de asbesto y la mortalidad media por mesotelioma para toda la población, estandarizada por edad, fue estimada en apenas 0,77/1.000.000 entre 1980 y $2010^{(10)}$. En el período de 2000 a 2012 , y limitándose a la población mayor de 30 años, este indicador fue de 2,1/1.000.000 $0^{(11)}$. Para cinco países de esta región, exclusivamente en el sexo masculino, la mayor mortalidad por mesotelioma fue para Chile $(3,1 / 1.000 .000)$, seguida por Argentina $(2,5 / 1.000 .000)$ y Uruguay $(2,3 / 1.000 .000)$, en tanto que Ecuador y Brasil tuvieron estimaciones semejantes $(0,5 / 1.000 .000)^{(12)}$. A partir de los resultados presentados, se observa que la mortalidad por mesotelioma en países de América Latina es menor que la de los países industrializados. Estas diferencias podrían deberse a la calidad y cobertura de los sistemas de información, además de variaciones del perfil productivo y de consumo, efectiva implementación de las medidas de protección, entre otras, y consecuentemente de la extensión e intensidad de la exposición ambiental u ocupacional al asbesto.

La mortalidad por mesotelioma es mayor en los hombres que en las mujeres. En la investigación de Delgermaa et al..$^{(5)}$, la tasa de mortalidad estandarizada por edad para todas las edades, entre 1994 y 2008, fue de $9,0 / 1.000 .000$ en hombres y 1,9/1.000.000 en mujeres, lo que corresponde a una razón hombre/mujer de 4,7/1. De modo similar, en Bélgica, la mortalidad estimada en hombres $(9,0 / 1.000 .000)$ fue mayor que la de las mujeres $(1,9 / 1.000 .000)^{(8)}$. Estos resultados refieren a la población general y son similares a los de la población de más de 40 años en Alemania, de 39,0/1.000.000 en hombres y de 8,0/1.000.000 para las mujeres, con una razón hombre/mujer de $4,8 / 1^{(13)}$. Se encontró una diferencia menor entre los sexos en 
España, donde la razón fue de 2,5/1 en 2010, atribuida a la menor presencia de la industria de asbesto en este país que comúnmente concentra a trabajadores del sexo masculino ${ }^{(14)}$, en forma similar a lo que ocurre en Bélgica( ${ }^{(8)}$. Esto denota la relevancia de la exposición ocupacional en hombres en comparación con la ambiental, común a ambos sexos ${ }^{(15)}$. Vale destacar que las mujeres y los niños también pueden estar afectados en forma indirecta por la exposición ocupacional al entrar en contacto con herramientas o vestimentas de los trabajadores expuestos ${ }^{(8)}$.

Según estudios realizados en Reino Unido $^{(16)}$ y EE.UU. ${ }^{(17)}$, la mortalidad por mesotelioma ha aumentado a partir de la década de 1950. Un análisis de datos de varios países ${ }^{(5)}$ mostró que la mortalidad por mesotelioma creció de 4,0/1.000.000 en 1994-2000 a 5,6/1.000.000 en 2001-2008, variación del $32,5 \%$ en el período y del $5,0 \%$ en promedio por año ${ }^{(5)}$. Un aumento un poco mayor $(39,9 \%)$ se encontró en España, donde la mortalidad aumentó de 2,8/1.000.000 en 1976-1980 a 3,9/1.000.000, en el período de 2006 a $2010^{(14)}$. Sin embargo, en Austria, entre 1970 y 2001, Neuberger y Vutuc ${ }^{(18)}$ estimaron la mortalidad por mesotelioma en 11,0/1.000.000, para el período 1970-1974, con una disminución a 8,4/1.000.000 entre 2000 y 2001, y una caída total del 24,0\%. En Brasil, único país de América Latina con publicaciones de series históricas, la tasa fue de 0,6/1.000.000 en 1980, con un crecimiento a 0,9/1.000.000 en 2010 , y un aumento del $46,9 \%{ }^{(10)}$. Vale señalar que en Gran Bretaña, las estimaciones oficiales muestran que la tasa de mortalidad por mesotelioma pasó de 3,3/1.000.000 en 1969-1971 a 39,9/1.000.000 en 2011-2013, lo que implica un aumento mayor al $1.000 \%$ a lo largo del período(7).

Esta tendencia puede reflejar el crecimiento de la prevalencia de expuestos al asbesto, así como características de la exposición, por ejemplo, la frecuencia, la intensidad y la duración, particularmente, en la ocupacional. De hecho, hay evidencias de que la prevalencia de expuestos ha disminuido entre los años 1970 y $2000^{(6,19)}$, pero su impacto sobre la mortalidad por mesotelioma solo se expresará después de 30 o 40 años, debido al largo período de latencia de este cáncer. Por un lado, se espera que el aumento de la mortalidad continue todavía por algunos años, lo que podría estar determinado por la presencia de asbesto en el ambiente ${ }^{(12)}$, el envejecimiento de la población ${ }^{(15)}$, un mayor acceso a recursos diagnósticos ${ }^{(13)} \mathrm{o}$ incluso a mejoras en los sistemas de información de mortalidad y en la Clasificación Internacional de la Enfermedades $(\mathrm{CIE})^{(8,13,19)}$. Por otro lado, en los países desarrollados, las mejoras en la calidad de los registros pueden explicar el aumento de la mortalidad a lo largo del tiempo, especialmente, cuando las estimaciones iniciales de mortalidad eran bajas ${ }^{(12)}$. Este incremento de la mortalidad ha sido descrito para los hombres y para las mujeres, aunque mayor para los primeros, lo que puede estar asociado a diferenciales de género desfavorables para los hombres en relación con las ocupaciones que se asocian a la exposición al asbesto(13).

En Argentina, entre 1994 y 2008, se identificaron 1.065 muertes por mesotelioma, con una media de 97 defunciones por año ${ }^{(20)}$. Otro estudio, para el periodo 1996-2007, presentó una tasa estandarizada por edad de la mortalidad por mesotelioma en hombres de 2,7/1.000.000, con un aumento del 8,9\% en este período ${ }^{(12)}$. Datos oficiales muestran que en el período subsiguiente, 2007-2011, 297 mujeres y 428 hombres fallecieron por mesotelioma, lo que corresponde a una tasa de mortalidad de $2 / 1.000 .000$ y $4 / 1.000 .000$, respectivamente, en ese periodo ${ }^{(21)}$.

Estos resultados son parciales. Para una adecuada vigilancia del mesotelioma, resultan necesarias series históricas más amplias y con otros descriptores. En este estudio se estima la tasa de mortalidad por mesotelioma y su distribución sociodemográfica y temporal en Argentina, para el periodo 1980-2013.

\section{MÉTODOS}

Se trata de un estudio de mortalidad por mesotelioma en Argentina para el período 
1980-2013, en individuos de 15 o más años de edad. La fuente de identificación de casos de mesotelioma fue el Sistema de Estadísticas Vitales, de la Dirección de Estadística e Información en Salud (DEIS) del Ministerio de Salud de la Nación, alimentado por los informes estadísticos de defunción. Los datos sobre la población de Argentina se obtuvieron de la base de datos del Instituto Nacional de Estadística y Censos (INDEC).

En este estudio, los casos de mesotelioma corresponden a los siguientes códigos:

- CIE-9 (1980-1996): 163.0 Neoplasia maligna de la pleura parietal, 163.1 Neoplasia de la pleura visceral, 163.8 Neoplasia maligna de otros sitios especificados de la pleural y 163.9 Neoplasia maligna de la pleura, parte no identificada.

- CIE-10 (1997-2013): C45.0 Mesotelioma de la pleura, C45.1 Mesotelioma del peritoneo, C45.2 Mesotelioma de pericardio, C45.7 Mesotelioma de otros sitios especificados y C45.9 Mesotelioma de sitio no especificado.

Solo se consideraron los diagnósticos de causa básica de muerte. Las variables descriptivas fueron sexo (masculino, femenino), edad y año de defunción.

Se calculó la tasa de mortalidad anual entre 1980 y 2013, dividiendo el número de defunciones por mesotelioma por el total de la población, con factor de multiplicación por 1.000 .000 para comodidad y facilidad de interpretación. Las tasas de mortalidad fueron estandarizadas por edad, para lo cual se utilizó el método directo según los estándares definidos por la Organización Mundial de Salud ${ }^{(22)}$ para la población mundial. Para los años no censales se calcularon las poblaciones mediante interpolación geométrica.

Los datos empleados son secundarios y públicos, y se encuentran protegidos por la Ley de Secreto Estadístico en Argentina (Ley 17622). El proyecto de investigación fue evaluado y aprobado por el Comité de Ética en Investigación del Instituto de Saúde Coletiva de la Universidade Federal da Bahia (CAAE 28218914.3.0000.5030).

\section{RESULTADOS}

Entre 1980 y 2013, se identificaron 3.259 defunciones por mesotelioma en Argentina. Con base en la CIE-9, empleada entre 1980-1996, fueron 1.279 casos, de los cuales la mayoría $(98,3 \%)$ fueron clasificadas con el código 163.9 Neoplasia maligna de la pleura, parte no especificada. En lo que resta del período, cuando se empleó la CIE-10, se encontraron 1.980 defunciones, cuyas causas de muerte también se concentraron en códigos no especificados (C45.9 Mesotelioma de sitio no especificado) en el $69,8 \%$ de los casos. A lo largo de este período, sin embargo, se observó una reducción de la proporción de diagnósticos no especificados, con relación al período anterior, en el que se usaba la CIE-9. Cabe señalar que en el período de uso de la CIE-10 (1997-2013), el nuevo código específico C45.0 Mesotelioma de la pleura correspondió al 22,1\% de los casos. Tampoco se detectaron diferencias de estos resultados entre hombres y mujeres (Tabla 1).

En 1980 se identificaron 64 defunciones, en tanto que, en 2013, este número se triplicó llegando a 177. El número de defunciones fue oscilante desde el año 1980 hasta 1997, a partir del cual se observa un aumento lineal de la tendencia (Figura 1). Ese patrón, que se repite entre los hombres, entre las mujeres inicia su aumento más tardíamente, en 2004. El aumento fue del 176,6\% para el total de defunciones, mayor entre los hombres $(189,7 \%)$ que entre las mujeres $(156 \%)$. En la Figura 2 se observa que las tasas de mortalidad, crudas y estandarizadas por edad, son semejantes, a lo largo de todo el período de estudio. En 1980, la mortalidad estandarizada por edad de mesotelioma fue de 3,3/1.000.000, y pasó a 2,3/1.000.0000 en 1997, período en el cual presentó oscilaciones. La tendencia de crecimiento lineal se hace clara a partir de1998 y hasta el final del período de estudio. Se observaron resultados similares en los cálculos de la tasa de mortalidad estandarizada por mesotelioma para cada sexo. Cabe señalar, que la mortalidad entre los hombres de 4,1/1.000.000 en 
Tabla 1. Distribución de las defunciones por mesotelioma en la población de 15 años o más, según el diagnóstico y sexo. Argentina, 1980-2013.

\begin{tabular}{|c|c|c|c|c|c|c|}
\hline \multirow[t]{2}{*}{ Códigos CIE } & \multicolumn{2}{|c|}{ Varones } & \multicolumn{2}{|c|}{ Mujeres } & \multicolumn{2}{|c|}{ Total } \\
\hline & $\mathrm{n}$ & $\%$ & $\mathrm{n}$ & $\%$ & $\mathrm{n}$ & $\%$ \\
\hline \multicolumn{7}{|l|}{ CIE-9 (1980-1996) } \\
\hline 163.0 NMP parietal & 6 & 0,8 & 2 & 0,4 & 8 & 0,6 \\
\hline 163.1 NMP visceral & 4 & 0,6 & 2 & 0,4 & 6 & 0,5 \\
\hline 163.8 NMP de otros sitios & 2 & 0,3 & 6 & 1,1 & 8 & 0,6 \\
\hline 163.9 NMP de parte no identificada & 702 & 98,3 & 555 & 98,1 & 1.257 & 98,3 \\
\hline Subtotal & 714 & 100,0 & 565 & 100,0 & 1.279 & 100,0 \\
\hline \multicolumn{7}{|l|}{ CIE-10 (1997-2013) } \\
\hline C45.0 Mesotelioma de la pleura & 264 & 21,9 & 171 & 22,1 & 435 & 22,0 \\
\hline C45.1 Mesotelioma de peritoneo & 23 & 1,9 & 17 & 2,2 & 40 & 2,0 \\
\hline C45.2 Mesotelioma de pericardio & 26 & 2,2 & 15 & 1,9 & 41 & 2,1 \\
\hline C45.7 Mesotelioma de otros sitios & 38 & 3,2 & 44 & 5,6 & 82 & 4,1 \\
\hline C45.9 Mesotelioma de sitio no especificado & 854 & 70,9 & 528 & 68,1 & 1.382 & 69,8 \\
\hline Subtotal & 1.205 & 100,0 & 775 & 100,0 & 1.980 & 100,0 \\
\hline
\end{tabular}

Fuente: Dirección de Estadísticas e Información en Salud (DEIS), Ministerio de Salud, Argentina. NPM = Neoplasia maligna de la pleura.

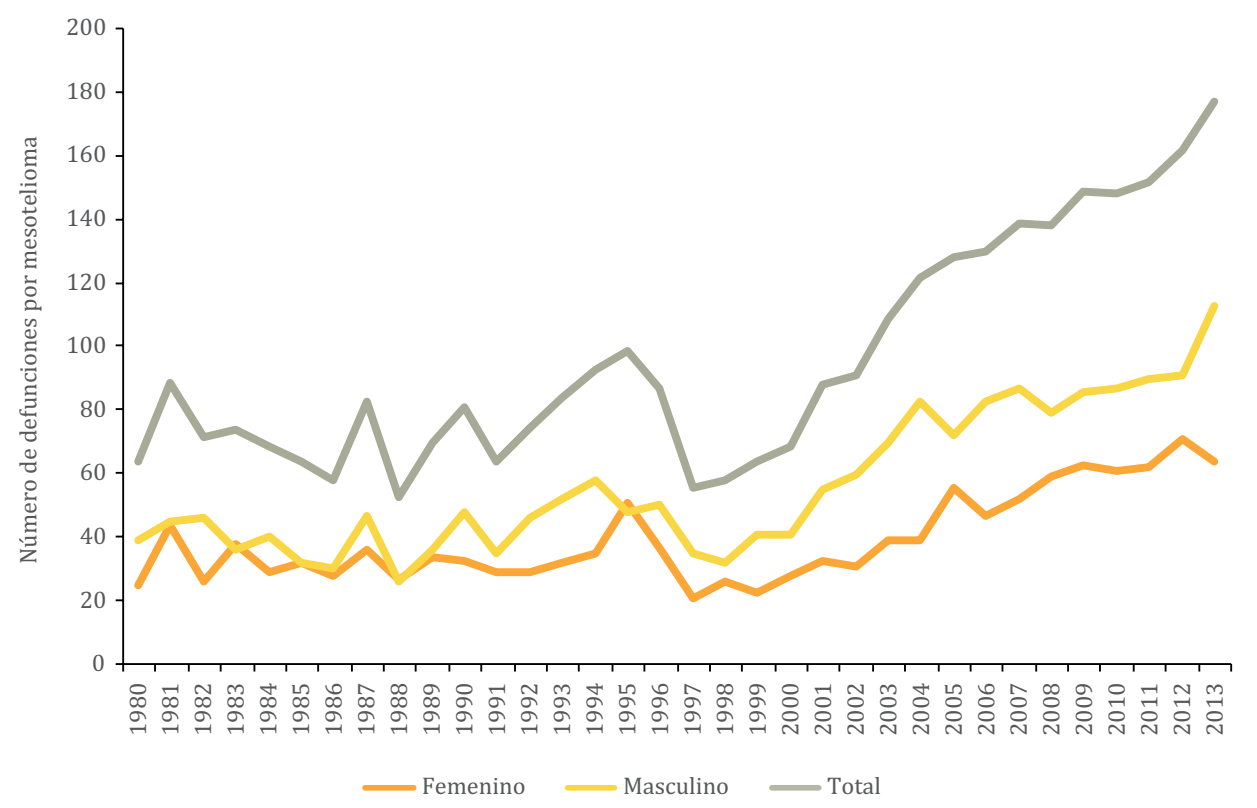

Figura 1. Distribución temporal de la mortalidad por mesotelioma en la población de 15 años o más, total y según sexo. Argentina, 1980-2013.

Fuente: Dirección de Estadísticas e Información en Salud (DEIS), Ministerio de Salud, Argentina. 


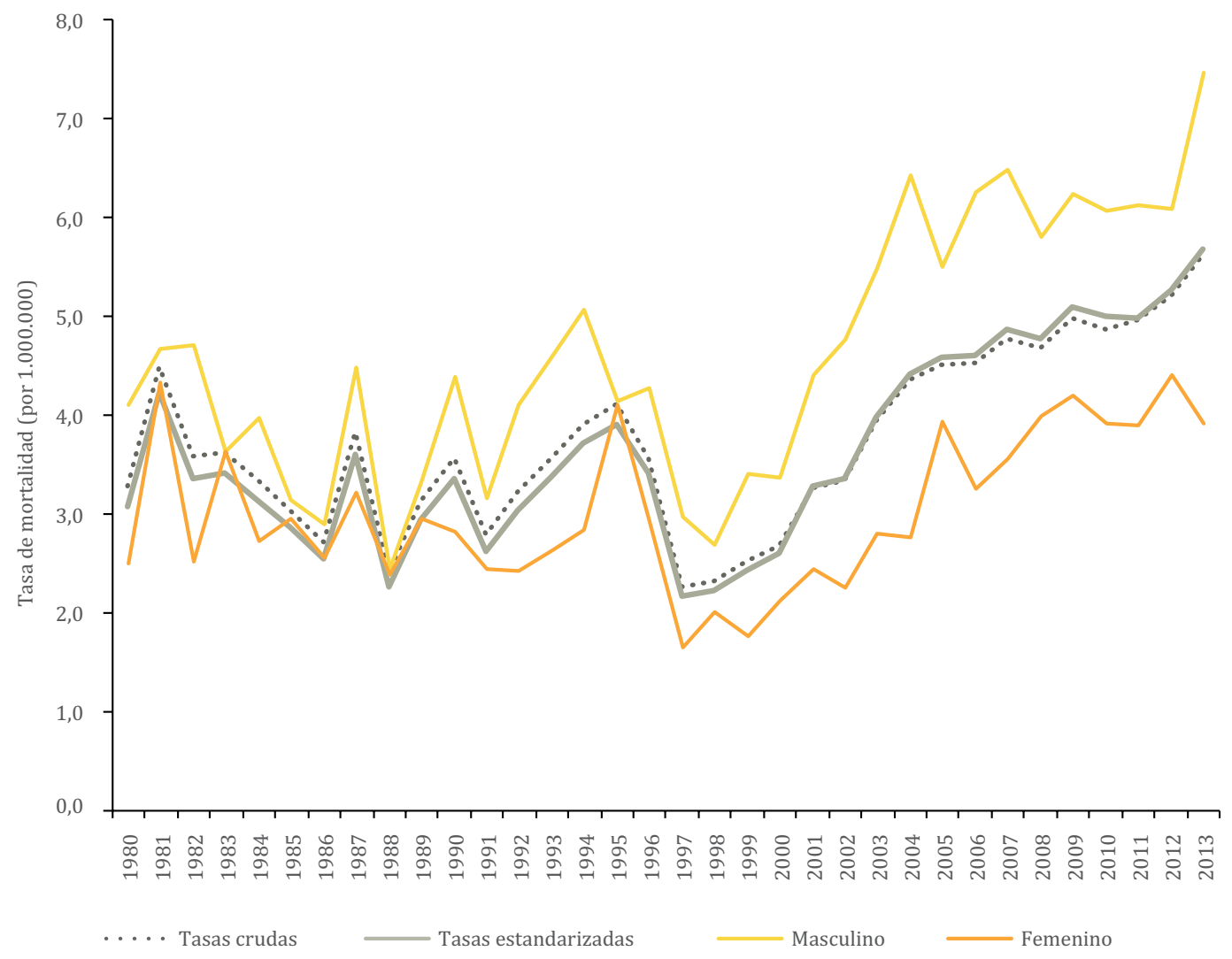

Figura 2. Distribución temporal de las tasas de mortalidad por mesotelioma, crudas y estandarizadas por edad, según sexo. Argentina, 1980-2013.

Fuente: Instituto Nacional de Estadística y Censos (INDEC), Dirección de Estadísticas e Información en Salud (DEIS), Ministerio de Salud, Argentina.

1980, cayó a 2,8/1.000.000 en 1998, y alcanzó el valor máximo de 7,5/1.000.000 en 2013. La mortalidad entre las mujeres, que fue de 2,5/1.000.000 en 1980, descendió a 1,7/1.000.000 en 1997, y osciló en el mismo nivel hasta 2004, cuando comenzó a crecer hasta llegar, en 2013, a 3,9/1.000.000.

\section{DISCUSIÓN}

Los resultados de este estudio muestran que en Argentina, entre 1980 y 2013, en la población de 15 años o más, el mesotelioma fue la causa básica de muerte de 3.259 personas, con registro de casos en todos los años. La mayor tasa de mortalidad fue de 5,7/1.000.000 en 2013 y la menor $(2,3 / 1.000 .000)$ en 1988. En el período de estudio se observó un aumento del número de defunciones de más del $170 \%$ y de la tasa de mortalidad por encima del $80 \%$. Este incremento de la tasa fue mayor entre los hombres $(81,9 \%)$ que entre las mujeres $(57,1 \%)$. La mayoría de los diagnósticos fueron clasificados con códigos descritos como "no especificados".

Los casos de mesotelioma indican que la exposición al asbesto siguió ocurriendo en el país, si se considera que la fracción atribuible de este cáncer a la exposición al asbesto se estima entre el $80 \%$ y el $90 \%{ }^{(19)}$. En Argentina, el asbesto se extrajo desde 1869 hasta la década de $1970^{(23)}$, y hay registros de importación de 21.206 toneladas en 1970, 
que descienden a 1 tonelada en 2002. No obstante, el consumo aparente, medido por la diferencia entre el volumen producido e importado se elevó desde 1995 hasta 2010, cuando alcanzó 341 toneladas. A partir de 2010, comenzó a caer hasta alcanzar 100 toneladas en 2013, último año de registro(24). No fueron encontrados datos de prevalencia de expuestos al asbesto, de tipo ocupacional individual o ambiental. El número de defunciones causadas por mesotelioma atribuible a la exposición ocupacional al asbesto fue de 735 para $2009^{(9)}$.

En este estudio, las tasas de mortalidad por mesotelioma fueron estimadas para la población general de 15 o más años de edad, a diferencia de otros estudios que consideraron la población total| ${ }^{(5,7,10)}$, o mayor de 30 años ${ }^{(11)}$. Aunque esto limita la comparabilidad, posibilita el cálculo de estimaciones más adecuadas considerando el bajo número de casos en los grupos de menor edad, además del largo período de latencia de 25 a 40 años. Con este límite de edad nuestras estimaciones de las tasas de mortalidad por mesotelioma en Argentina fueron mayores aunque quedaron por debajo de las relatadas para otros países calculadas con referencia al total de la población ${ }^{(20)}$. Los valores menores de las tasas de mortalidad por mesotelioma en Argentina pueden ser resultantes de bajos niveles de exposición: son pocas las fuentes naturales de asbesto, y el volumen de producción e importación ha sido pequeño en relación con otros países. Otra explicación posible podría derivarse de la complejidad técnica y de los costos para realizar un diagnóstico adecuado del mesotelioma, dado que requiere de un entrenamiento específico de los médicos para su evaluación. Esta problemática fue identificada y debatida durante la IV Semana Argentina de la Salud y la Seguridad en el Trabajo en $2007^{(25)}$. Nuestros hallazgos fueron mayores que las estimaciones disponibles para los países de la región como Brasil(10), lo que contrasta con la mayor producción, uso y consumo de asbesto ${ }^{(26)}$. La OMS clasifica la calidad de los registros de muertes en Argentina como de buena calidad para su uso en la vigilancia en salud ${ }^{(27)}$.
Los hombres tuvieron mayor riesgo de morir por mesotelioma que las mujeres, en cada uno de los años considerados, resultado similar al encontrado en otros países ${ }^{(5,8,13,18,28)}$. Esa diferencia parece resultar de la mayor prevalencia de exposición ocupacional al asbesto entre los hombres, que se concentran en las actividades de extracción, fabricación de productos y la construcción, entre otras ${ }^{(8)}$. Los estudios muestran que el riesgo de muerte por mesotelioma es mayor cuando la exposición es ocupacional, comúnmente de mayor intensidad que la ambiental ${ }^{(29)}$. En Argentina, los datos del perfil productivo general muestran una predominancia de hombres en las ocupaciones que habitualmente se asocian al contacto con el asbesto ${ }^{(30)}$.

En este estudio, las tasas de mortalidad por mesotelioma aumentaron entre $1980 \mathrm{y}$ 2013, en general y para hombres y mujeres, por separado. Esta tendencia es sugerente de un aumento histórico de la frecuencia, intensidad y/o duración de la exposición, así como de la prevalencia de expuestos al asbesto. Más allá de esto, el tiempo de latencia de este cáncer es muy largo. La oscilación de la mortalidad entre 1980 y 1997 puede estar provocada por las dificultades para realizar el diagnóstico y su codificación, por entonces basados en la CIE-9, que solo incluía la neoplasia de la pleura, sin especificar el mesotelioma ${ }^{(8)}$. Las oscilaciones en las estimaciones de mortalidad son comunes para enfermedades raras debido a los pequeños números con que se trabaja. La tendencia de crecimiento observada a partir de 1998 puede también ser el efecto de la incorporación del diagnóstico específico "mesotelioma" en la CIE 10 y también en la calidad y cobertura del sistema de registro de defunciones en Argentina ${ }^{(31)}$. En países con bajas tasas de mortalidad por mesotelioma como Argentina, los aumentos en las series históricas pueden ser el resultado de estos avances ${ }^{(5)}$, aunque un verdadero incremento del riesgo no puede descartarse, considerando que patrones similares han sido observados en otros países ${ }^{(5)}$ con prohibición más reciente del asbesto. Los datos sobre producción, importación y consumo de asbesto en Argentina son 
incompletos ${ }^{(24)}$, lo que limita la comprensión de su papel para la explicación de estas tendencias. No se encontraron datos sobre la exposición al asbesto entre trabajadores, considerada como la que tiene mayor contribución al mesotelioma.

El mayor aumento de la mortalidad por mesotelioma en los hombres respecto de las mujeres, a lo largo del tiempo, parece reflejar diferencias en el crecimiento de las actividades económicas asociadas al asbesto, que cuenta con mayor participación de trabajadores del sexo masculino. Esto también sugiere un mayor crecimiento de la exposición ocupacional, caracterizada por mayores frecuencias, intensidades y duración en los ambientes ocupacionales en relación con la ambiental que afecta, independientemente, a ambos sexos.

Los hallazgos de este estudio pueden estar afectados por algunas limitaciones metodológicas. Por ejemplo, los ya mencionados problemas en el diagnóstico y registro debido a la complejidad y costo de los procedimientos implicados ${ }^{(32)}$. El diagnóstico de mesotelioma según las consideraciones del protocolo propuesto por el Finnish Institute of Occupational Health ${ }^{(33)}$, requiere el entrenamiento y conocimiento por parte de los profesionales de la salud sobre la importancia de este diagnóstico, no solo para el tratamiento, sino también para la prevención, dado que se trata de una enfermedad evitable ${ }^{(34)}$. Es posible que las empresas hayan presionado para no registrar los casos de sus trabajadores debido a intereses económicos como en otros países ${ }^{(35)}$. En un estudio se presentaron estimaciones de subregistro del $20 \%$ al $25 \%$ para las defunciones por mesotelioma en el mundo ${ }^{(21)}$.

En Argentina, no se encontraron estimaciones del subregistro de muertes por mesotelioma, aunque el sistema de información sobre mortalidad ha sido clasificado como de "buena" calidad (25). Aun así, la mayoría de los diagnósticos tanto de la CIE-9 como de la CIE-10 fueron no especificados, lo que puede estar indicando la dificultad de establecer si este tumor es de origen primario o metastásico. La razón hombre/mujer de la mortalidad por mesotelioma en este estudio fue menor que la encontrada en otros países, lo que puede deberse a la subenumeración diferencial entre los sexos. Esto es posible, si se considera la elevada carga de exposición ocupacional al asbesto para el mesotelioma en el sexo masculino ${ }^{(29)}$.

Es necesario que se realicen estudios sobre la distribución de la mortalidad por mesotelioma entre los grupos ocupacionales y ramos de actividad económica, así como el mapeo de fuentes de exposición ocupacional y ambiental, fundamentales para la prevención. Este es uno de los primeros estudios de ámbito nacional sobre el mesotelioma en Argentina con una serie histórica de mortalidad, que puede servir de motivación para que se realicen otros estudios de vigilancia de cáncer ocupacional. Estos resultados proveen evidencias de interés para gestores y tomadores de decisiones, especialmente en la implementación efectiva de la vigilancia en salud, con foco en el asbesto y sus efectos sobre la salud. Esto supone acciones sucedáneas a la prohibición, que por sí solas no aseguran la eliminación de la exposición. Para esto, es necesario acompañar la implementación de la prohibición del uso, extracción e importación, con la remoción segura de sus productos y el adecuado destino de los desechos. Además de esto, las políticas que faciliten la disponibilidad de materiales alternativos al asbesto para uso industrial pueden favorecer la prevención de la exposición.

La difusión de estas informaciones puede contribuir a dar mayor visibilidad a este problema de salud colectiva, especialmente, para profesionales de la salud y de organizaciones de trabajadores y actores sociales comprometidos con la lucha contra este cancerígeno. También, el acceso de este tipo de información por parte de los colectivos de trabajadores podría ser empleado como base para jerarquizar y valorizar el derecho a la información e insistir en las reivindicaciones por mejores condiciones de seguridad en el trabajo. 


\section{AGRADECIMIENTOS}

El primer autor recibió una beca de doctorado del Conselho Nacional de Desenvolvimento Cientifico e Tecnológico (CNPq) de 2012 a 2013 y de la Coordenação de Aperfeiçoamento de Profissionais do Ensino Superior (CAPES) de 2013 a 2015. La segunda autora es becaria de productividad $1 \mathrm{C}$ del CNPq. Este trabajo fue elaborado como parte de la cooperación entre el Programa de Pós-Graduação em Saúde Coletiva, del Instituto de Saúde Coletiva, Universidade Federal da Bahia (ISC-UFBA), y el Instituto de Salud Colectiva de la Universidad Nacional de Lanús (ISCo-UNLa).

\section{REFERENCIAS BIBLIOGRÁFICAS}

1. International Agency for Research on Cancer. Arsenic, metals, fibres, and dusts: Volume $100 \mathrm{C}$, A review of human carcinogens [Internet]. Lyon: IARC, WHO; 2012 [citado 15 jun 2016]. Disponible en: http://tinyurl.com/az5hd48.

2. World Health Organization. Chrysotile asbestos. [Internet]. 2014 [citado 1 sep 2016]. Disponible en: http://tinyurl.com/z9f5u55.

3. Secretaría de Ambiente y Desarrollo Sustentable de la Nación. Primer compendio de estadísticas ambientales: República Argentina. Buenos Aires: Secretaría de Ambiente y Desarrollo Sustentable de la Nación; 2008.

4. Weill H, Hughes J, Churg A. Changing trends in US mesothelioma incidence. Occupational and Environmental Medicine. 2004;61(5):438-441.

5. Delgermaa V, Takahashi K, Park E, Vinh G, Toshiyuki H, Sorahan T. Global mesothelioma deaths reported to the World Health Organization between 1994 and 2008. Bulletin of the World Health Organization. 2011;89(10):716-724.

6. Kameda T, Takahashi K, Kim R, Jiang Y, Movahed M, Park E, Rantanen J. Asbestos: use, bans and disease burden in Europe. Bulletin of the World Health Organization. 2014;92:790-797.

7. Health and Safety Executive. Mesothelioma in Great Britain: Mesothelioma mortality in Great Britain 1968-2013 [Internet]. London: HSE; 2014 [citado 07 jun 2016] Disponible en: http://tinyurl. com/d5fa93z.

8. Van den Borre L, Deboosere P. Asbestos in Belgium: an underestimated health risk: The evolution of mesothelioma mortality rates (1969-2009). International Journal of Occupational and Environmental Health. 2014;20(2):134-140.
9. Passeto R, Terracini B, Marsili D, Comba P. Occupational burden of asbestos-related: Cancer in Argentina, Brazil, Colombia, and Mexico. Annals of Global Health. 2014;80:263-268.

10. Pedra F, Oliveira da Silva P, Mattos I, Castro H. Mesothelioma mortality rate in Brazil, 1980 to 2010. Revista Brasileira de Cancerologia. 2014;60(3):199-206.

11. Algranti E, Saito C, Carneiro A, Moreira B, Mendoça E, Bussacos M. The next mesothelioma wave: Mortality trends and forecast to 2030 in Brazil. Cancer Epidemiology. 2015;39(5):687-692.

12. Nishikawa K, Takahashi K, Karjalainen A, Wen C, Furuya S, Hoshuyama T, Todoroki M, Kiyomoto Y, Wilson D, Higashi T, Ohtaki M, Pan G, Wagner G. Recent mortality from pleural mesothelioma, historical patterns of asbestos use, and adoption of bans: a global assessment. Environmental Health Perspectives. 2008;116(12):1675-1680.

13. Schonfeld SJ, McCormack V, Rutherford MJ, Schuz J. Regional variations in German mesothelioma mortality rates: 2000-2010. Cancer Causes and Control. 2014;25(5):615-624.

14. López-Abente G, García-Gómez M, MenéndezNavarro A, Fernández-Navarro P, Ramis R, GarcíaPérez J, Cervantes $M$, Ferreras $E$, Jiménez-Muñoz $M$, Pastor-Barriuso R. Pleural cancer mortality in Spain: time-trends and updating of predictions up to 2020. BMC Cancer. 2013;13:528.

15. Pitarque $S$, Clèries $R$, Martínez JM, LópezAbente G, Kogevinas M, Benavides FG. Mesothelioma mortality in men: trends during 1977-2001 and projections for 2002-2016 in Spain. Occupational and Environmental Medicine. 2008;65(4): 279-282.

16. Peto J, Hodgson JT, Matthews FE, Jones JR. Continuing increase in mesothelioma mortality in Britain. Lancet. 1995;345(8949):535-539.

17. Price B, Wave A. Mesothelioma trends in the United States: an update based on surveillance, epidemiology, and end results program data for 1973 through 2003. American Journal of Epidemiology. 2004;159(2):107-112.

18. Neuberger $M$, Vutuc $C$. Three decades of pleural cancer and mesothelioma registration. International Archives of Occupational and Environmental Health. 2003;76:161-166.

19. Stayner L, Welch LS, Lemen R. The worldwide pandemic of asbestos-related diseases. Annual Review of Public Health. 2013;34:205-216. 
20. Park EK, Takahashi K, Hoshuyama T, Cheng TJ, Delgermaa V, Le GV, Sorahan T. Global magnitude of reported and unreported mesothelioma. Environmental Health Perspectives. 2011;119(4):514-518.

21. Instituto Nacional del Cáncer, Ministerio de Salud de la Nación. Atlas de mortalidad por cáncer en Argentina, 2007-2011. Buenos Aires: Ministerio de Salud de la Nación; 2013.

22. Ahmad OB, Boschi-Pinto C, Lopez AD, Murray CJL; Lozano R, Inoue M. Age standardization of rates: a new WHO standard (GPE Discussion Paper Series: No. 31) [Internet]. WHO; 2001 [citado 15 jun 2016]. Disponible en: http:// tinyurl.com/jl9vak9.

23. Rodriguez E. Asbestos Banned in Argentina. International Journal of Occupational and Environmental Health. 2004;10(2):202-208.

24. Virta RL. Worldwide asbestos supply and consumption trends from 1900 through 2003: Circular 1298. Reston, Virginia: U.S. Geological Survey; 2006.

25. McCulloch J, Geoffrey-Tweedale G. Defending the indefensible: The global asbestos industry and its fight for survival. New York: Oxford University Press; 2008.

26. Capone L, Consiglio E, Fulgenci AE, Jarés E, Labbate A, Poropat A. Patología respiratoria de origen ocupacional: Ateneo SRT-AAMR. Buenos Aires: Superintendencia de Riesgos del Trabajo; 2007.

27. Marsili D, Terracini B, Santana VS, Ramos-Bonilla JP, Pasetto R, Mazzeo A, Loomis D, Comba $\mathrm{P}$, Algranti E. Prevention of asbestos-related disease in countries currently using asbestos. International Journal of Environmental Research and Public Health. 2016;13(5): E494.
28. McElveny DM, Darnton AJ, Price MJ, Hodgson JT. Mesothelioma mortality in Great Britain from 1968 to 2001. Occupational Medicine. 2005;55:79-87.

29. Lacourt A, Gramond C, Rolland P, Ducamp S, Audignon S, Astoul P, Chamming's S, GilgSoitllg A, Rinaldo M, Raherison C, Galateau-Salle F, Imbernon E, Pairon JC, Goldberg M, Brochard P. Occupational and non-occupational attributable risk of asbestos exposure for malignant pleural mesothelioma. Thorax 2014;69(6):532-539.

30. Superintendencia de Riesgos del Trabajo. Informe sobre indicadores de accidentabilidad por provincia y sector económico: Procesamiento especial-año 2006. Buenos Aires: Instituto de Estudios Estratégicos y Estadísticas, Área de Estadísticas Superintendencia de Riesgos del Trabajo; 2006.

31. Alazraqui $M$, Spinelli $H$, Zunino MG, Souza ER. Calidad de los sistemas de información de mortalidad por violencias en Argentina y Brasil - 1990-2010. Ciência e Saúde Coletiva. 2012;17(12):3279-3288.

32. Addis B, Roche H. Problems in mesothelioma diagnosis. Histopathology. 2009;54(1):55-68.

33. Finnish Institute of Occupational Health. Asbestos, asbestosis, and cancer Helsinki criteria for diagnosis and attribution 2014. Helsinki: Finnish Institute of Occupational Health; 2014.

34. Collegium Ramazzini. Asbestos is still with us: repeat call for a universal ban. New Solut. 2010;20(2):257-66.

35. Pan American Health Organization. Health information and analysis: Health situation in the Americas: Basic indicators 2008. Washington DC: PAHO; 2008. 